Original Article

\title{
Predictive validity of the functional independence and difficulty scale in community-dwelling Japanese older adults
}

\author{
Takashi Saito, RPT, $\mathrm{PhD}^{1)^{*}}$, Nobuko Matsui, RPT, MS ${ }^{2)}$, Shuichiro Watanabe, MD, PhD ${ }^{3)}$ \\ 1) Visiting Nursing Station Happiness Care: 1-24-27-201 Takadanobaba, Shinjuku-ku, Tokyo, Japan \\ 2) Department of Physical Therapy, Tokyo College of Allied Medicine, Japan \\ 3) Graduate School of Gerontology, J. F. Oberlin University, Japan
}

\begin{abstract}
Purpose] The newly developed Functional Independence and Difficulty Scale is a tool for assessing the performance of basic activities of daily living in terms of both independence and difficulty. The aim of this study was to examine the predictive validity of the scale for decline of instrumental activities of daily living ability and multiple falls during a 24-month follow-up period. [Subjects and Methods] One-hundred forty older adults (median age $74.0,60 \%$ women) completed baseline data collection and a follow-up postal survey. At baseline, background variables, the Functional Independence and Difficulty Scale, and instrumental activities of daily living ability assessed by the five sub-items of Tokyo Metropolitan Institute of Gerontology Index of Competence were recorded. At follow-up, data on instrumental activities of daily living ability and falls in the previous 12 months were obtained. [Results] Of the 140 participants, 15 (10.7\%) declined in instrumental activities of daily living ability and $14(10.0 \%)$ experienced multiple falls. The Functional Independence and Difficulty Scale at baseline independently predicted decline of instrumental activities of daily living ability and multiple falls. [Conclusion] The Functional Independence and Difficulty Scale predicts subsequent decline of instrumental activities of daily living ability and multiple falls.

Key words: Basic activities of daily living, Instrumental activities of daily living, Predictive validity
\end{abstract}

(This article was submitted Jan. 19, 2017, and was accepted Feb. 20, 2017)

\section{INTRODUCTION}

Basic activities of daily living (BADL), which typically include bathing, toileting, dressing, transferring, and feeding, are a critical aspect of disability ${ }^{1)}$. Thus, BADL rating scales are widely used as a measure of functional ability in clinical practice of physical therapy and community studies with older people. BADL scales are used for descriptive purposes ${ }^{2)}$, observing changes over time ${ }^{3}$, judging the effect of intervention ${ }^{4)}$, and predicting future adverse health events ${ }^{5,6)}$ for both clinical practice and research.

Although BADL disability can be defined as dependence, i.e., "requiring help from another person" or difficulty, i.e., "the report of any difficulty as a result of a health or physical problem" with a BADL task ${ }^{7}$, Gill and colleagues ${ }^{6}$ ) emphasized the usefulness of a BADL scale for assessing both independence and difficulty. Using data from cross-sectional and longitudinal analyses, they found that community-dwelling older adults who had independent BADL performance with perceived difficulty had functional profiles, physical performance scores, and rates of healthcare utilization and death that were intermediate compared to those of older adults who had independent BADL performance without perceived difficulty and those who had dependent BADL performance ${ }^{6}$. These findings imply that older adults who were BADL independent with difficulty are more frail than are those without difficulty, suggesting that they are a population at high risk for adverse health events. To

*Corresponding author. Takashi Saito (E-mail: takashi-riha@live.jp)

(C2017 The Society of Physical Therapy Science. Published by IPEC Inc.

This is an open-access article distributed under the terms of the Creative Commons Attribution Non-Commercial No Derivatives (by-nc-nd) License. (CC-BY-NC-ND 4.0: http://creativecommons.org/licenses/by-nc-nd/4.0/) 
capture these older adults and evaluate their BADL disability, scales assessing either BADL dependency or difficulty would be inadequate. Scales that can assess both BADL independence and difficulty provide complementary information about disability ${ }^{6}$.

Thus, in our previous studies ${ }^{8-11)}$, we have developed a new BADL assessment tool (Functional Independence and Difficulty Scale; FIDS) that can assess BADL ability as both performance and perceived difficulty among community-dwelling older people. The FIDS has 14 items on BADL. The function scores for the FIDS range from 14 to 42, with higher scores representing better function as detailed in our previous report ${ }^{8}$. One of the features of FIDS is that the scale can detect BADL perceived difficulty among older adults performing BADL independently.

A useful measure should show high reliability (i.e., relatively free of random error) and be valid (i.e., measure what it is supposed to measure $)^{12)}$. Therefore, reliability and validity are desirable measurement properties for the newly developed FIDS. Moreover, many different methods to assess BADLs have been developed and described ${ }^{13)}$. Because there are many existing measures, it is important to know whether the newly developed FIDS offers added benefit over these measures.

Our recent studies ${ }^{8-11)}$ have shown that the FIDS is easy to administer and is a reliable and valid measure for assessing BADL ability of community-dwelling older people. The FIDS shows acceptable item validity ${ }^{8)}$, internal consistency ${ }^{8)}$, and test-retest and inter-rater reliability ${ }^{10}$. The concurrent validity of the FIDS was also demonstrated through its correlation with existing BADL measures ${ }^{8,9)}$, health-related quality of life (HRQOL) ${ }^{9)}$, and physical function ${ }^{11)}$. Moreover, the FIDS shows a relatively small ceiling effect compared to the existing BADL assessment, the Barthel index (BI $)^{14)}$, in healthy and frail older Japanese adults ${ }^{9)}$.

Because these factors that are correlated with the FIDS (HRQOL and physical function) predict adverse health events, such as decline of function ${ }^{15)}$ and falls ${ }^{16)}$, the FIDS may also predict these adverse health events. However, the cross-sectional nature of previous studies ${ }^{8-11)}$ does not allow strict cause-effect interpretations of the associations between the FIDS and adverse events. Consequently, the predictive validity of the FIDS for adverse health events in the long term has not yet been established. Therefore, using a longitudinal study design, the aim of this study was to assess the predictive validity of the FIDS for adverse health events.

We initially hypothesized that the FIDS would predict subsequent decline of instrumental activities of daily living (IADL) ability and falls in community-dwelling Japanese older adults. Our second hypothesis was that the FIDS would show better predictive ability in estimating these adverse health events than would the existing BADL scale assessing BADL dependency. These two hypotheses were based on the features of the FIDS: the scale can capture information about both dependency and difficulty of BADLs and can detect frail older adults among those who can perform BADLs independently.

To test these hypotheses, we focused on the $\mathrm{BI}^{14)}$ and compared the predictive ability for subsequent decline of IADLs and multiple falls between the FIDS and BI. The BI assesses BADL dependency and is a representative existing BADL measure that has been recommended, together with the Functional Independence Measure ${ }^{17)}$, as a measure of activity ${ }^{18)}$, and has been proposed as the standard index for clinical and research purposes ${ }^{19)}$.

\section{SUBJECTS AND METHODS}

All procedures performed in studies involving human participants were in accordance with the ethical standards of the institutional and/or national research committee and with the 1964 Declaration of Helsinki and its later amendments or comparable ethical standards. The study protocol was approved by the ethics Committee of J. F. Oberlin University (approval no. 14049). Informed consent was obtained from all individual participants included in the study. This study was supported by the Univers Foundation (grant number 15-02-144).

This study was a longitudinal study. Recruitment was conducted using convenience sampling. All participants in this longitudinal study were enrolled from Tsumagoi district, Gunma Prefecture, Japan. Tsumagoi district is a rural area located about $150 \mathrm{~km}$ north of Tokyo. The participants were individuals who voluntarily joined a specific health examination provided to those insured by public medical insurance. At the public hall, individuals were invited to participate in this study by the researchers.

We applied the following inclusion criteria: aged 65 years and over, living in the community, being able to respond to the questionnaire in Japanese, and not using Japanese long-term care services ${ }^{20)}$. The exclusion criterion was subjects who were blind. Subjects who did not match these criteria or those who did not want to participate in research procedures voluntarily were excluded.

Baseline data collection was carried out on April 2014. Each subject answered the questionnaire by themselves, and the answers were checked by an examiner. When self-administration of the questionnaire was difficult for any reason, the examiner interviewed the subject. A follow-up postal survey was conducted 24 months after baseline data collection (April 2016), with an offer of assistance in completing the study.

In this prospective study, we included participants who completed baseline data collection and follow-up postal survey. Of the participants recruited in the baseline data collection session $(\mathrm{N}=252)$, one candidate was blind, four refused to participate, and 22 had missing data. Thus, 225 participants at baseline were included in the current prospective study.

Background variables included age, gender, height, weight, and degree of independence of daily living. Criteria assessing the degree of independence of daily living were as follows: independent, going outside independently; house-bound, needing 
help to go outside but, in general, living independently within their house; and bedbound, needing help for all BADLs.

The FIDS assesses BADL ability as performance and perceived difficulty ${ }^{8}$. The FIDS comprises 14 BADL-related items: getting up from bed, standing up from a chair, standing up from the floor, dressing, putting on pants, eating, cleaning after toileting, washing, brushing teeth, opening a polyethylene terephthalate (PET) bottle, cutting toenails, walking inside, walking outside, and going up and down 4-6 steps. The FIDS has two questions for each item, one question about independence (A) and one about perceived difficulty (B). The response to each question was designed as "yes" (A: needing help/unable, B: having difficulty in doing) or "no" (A: needing no help, B: having no difficulty). Scores were assigned as follows: 1 if the participant reported being dependent or unable to perform the activity (response to question A is "yes"), 2 if independence with difficulty was reported (response to question A is "no" and response to question B is "yes"), and 3 if independence without difficulty was reported (response to questions A and B are both "no"). The function scores for the FIDS range from 14-42, with higher scores representing better function.

The BI includes 10 items of BADLs, as detailed in the original report ${ }^{14)}$. This scale ranges from 0 to 100 , where higher scores are associated with a greater degree of independence.

In a follow-up postal survey, we collected data regarding IADL ability and falls as outcome measures. IADL ability was assessed using the five sub-items of Tokyo Metropolitan Institute of Gerontology Index of Competence (TMIG-IC) ${ }^{21)}$. The TMIG-IC was designed to evaluate capacity higher than BADL and consists of three subscales: instrumental self-maintenance (5 items), intellectual activity (4 items) and social role (4 items) $)^{21)}$. The response to each item is simply "yes" (able to do) or "no" (unable), and scored 1 for "yes" and 0 for "no." The total score is the sum of all 13 items, with a higher score (maximum 13 points) indicating higher competence of the elderly. In our study, the instrumental self-maintenance subscale, with a higher score (maximum 5 points) indicating higher ability, was used to assess IADL ability. The five sub-items are as follow: (1) "Can you use public transportation (bus or train) by yourself?", (2) "Are you able to shop for daily necessities?", (3) "Are you able to prepare meals by yourself?", (4) "Are you able to pay bills?", and (5) "Can you handle your own banking?" In this study, "subsequent decline of IADL ability" was defined as decline in the instrumental self-maintenance sub-score of at least 1 point compared to baseline.

Fall frequency in the previous 12 months was measured at the follow-up postal survey, 24 months after baseline data collection. On the follow-up postal survey questionnaire, participants were asked "How many falls did you have in the past year?" A previous study ${ }^{22}$ showed that multiple fallers typically have poor health states, balance, or overall cognitive functions as compared to single fallers, but single fallers did not differ from non-fallers. Moreover, multiple fallers experience greater substantial decline than do single fallers in IADL/BADL and social functions ${ }^{23}$. Thus, it is important to pay attention to multiple falls in clinical practice and research on elderly individuals. In this study, "fallers" were defined as people who had multiple falls, defined as two or more falls, during the previous 12 months.

Statistical analyses were carried out using IBM SPSS Statistics (Version 22, IBM Japan Ltd.). A p-value of $<0.05$ was considered indicative of statistical significance. Continuous variables are reported as mean \pm standard deviation (SD) or median (interquartile range, IQR), and categorical variables are reported as numbers and percentages to describe the samples and provide summary information regarding the measures used. We compared the baseline characteristics of participants between those with and without adverse outcomes at follow-up. Unpaired Mann-Whitney and $\chi^{2}$ tests were used.

Logistic regression analysis was performed to examine whether the FIDS and BI were independently associated with adverse outcome measures. In this analysis, subsequent decline of IADL ability and multiple falls was included as a dependent variable, and age, gender, FIDS, and BI were included as independent variables. To compare the predictive ability for outcome measures between the FIDS and BI, logistic regression analysis was performed using two models. Model 1 included age, gender, and BI as independent variables, and model 2 included age, gender, and FIDS as independent variables. Odds ratios (ORs) and 95\% confidence intervals (CIs) were calculated for the outcome measures.

\section{RESULTS}

A total of 225 participants at baseline were included in the current prospective study. Of those, 51 were excluded because of non-response and 34 because of missing data. Ultimately, 140 participants (ratio of valid responses; $62.2 \%$ ) were included in analysis (Fig. 1). There were no statistically significant differences in participant characteristics between those excluded and those included in the analysis (the final sample), except for age (median 78.0 years (IQR, 72.0-83.0) in excluded versus 74.0 years (IQR, 70.0-80.0) in included, $\mathrm{p}=0.02$ ) and total TMIG-IC score (median 13.0 points (IQR, 11.0-13.0) in excluded versus 13.0 points (IQR, 12.0-13.0) in included, $\mathrm{p}=0.005$ ).

Table 1 shows characteristics, FIDS, BI, and TMIG-IC at the baseline assessment. Overall sample of 140 had a median age of 74.0 years; $60 \%$ were women. All participants satisfied the criteria of degree of independence of daily living as "independent," meaning going outside independently. There were significant differences in age, height, weight, and FIDS score between men and women. Women were significantly younger $(\mathrm{p}<0.05)$, smaller $(\mathrm{p}<0.001)$ and lighter $(\mathrm{p}<0.001)$. Moreover, women scored lower on the FIDS than men $\operatorname{did}(\mathrm{p}<0.05)$.

The baseline characteristics of those with and without adverse events are shown in Table 2. At the 24-month follow-up postal survey, 15 participants $(10.7 \%)$ declined in their score on the instrumental self-maintenance subscale on the TMIG-CI, and 14 participants $(10.0 \%)$ experienced at least two falls. When comparing the baseline score on the FIDS between those 


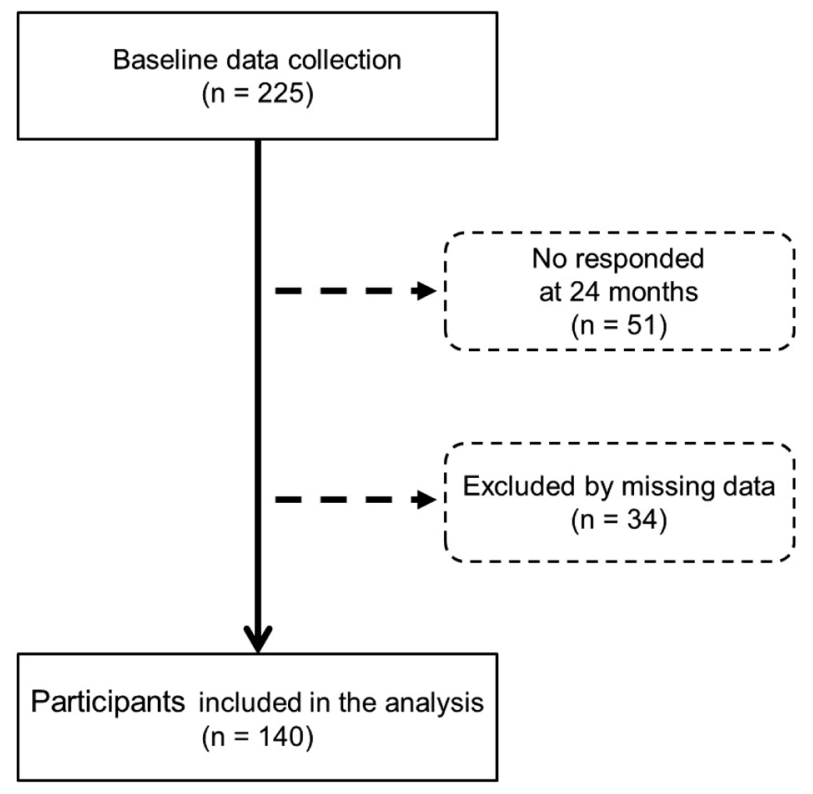

Fig. 1. Flow of study participants

Table 1. Baseline characteristics of the participants and results of comparison between men and women

\begin{tabular}{|c|c|c|c|c|}
\hline & $\begin{array}{c}\text { Total } \\
(\mathrm{n}=140)\end{array}$ & $\begin{array}{c}\text { Men } \\
(\mathrm{n}=56)\end{array}$ & $\begin{array}{l}\text { Women } \\
(\mathrm{n}=84)\end{array}$ & \\
\hline Age (years) & $74.0(70.0-80.0)$ & $76.5(71.0-82.0)$ & $73.0(69.5-79.0)^{*}$ & $\mathrm{a}$ \\
\hline Height (cm) & $153.3(148.3-159.9)$ & $160.1(156.1-164.8)$ & $150.2(146.3-153.3)^{* *}$ & a \\
\hline Weight (kg) & $55.7(48.8-62.4)$ & $59.9(55.8-66.4)$ & $52.4(47.5-57.2)^{* *}$ & $\mathrm{a}$ \\
\hline BMI $\left(\mathrm{kg} / \mathrm{m}^{2}\right)$ & $23.2(21.6-25.5)$ & $23.5(21.6-25.4)$ & $23.2(21.5-25.5)$ & $\mathrm{a}$ \\
\hline \multicolumn{5}{|c|}{ Independence in daily living, $\mathrm{n}(\%)$} \\
\hline Independent & $140(100)$ & $56(100)$ & $84(100)$ & \multirow{2}{*}{ - } \\
\hline House-bound/Bed-bound & $0(0)$ & $0(0)$ & $0(0)$ & \\
\hline Total BI score (points) & $100.0(100.0-100.0)$ & $100.0(100.0-100.0)$ & $100.0(100.0-100.0)$ & $\mathrm{a}$ \\
\hline \multirow[t]{2}{*}{ Total FIDS score (points) } & $42.0(41.0-42.0)$ & $42.0(41.0-42.0)$ & $42.0(41.0-42.0)^{*}$ & \multirow[b]{2}{*}{$\mathrm{a}$} \\
\hline & $41.1 \pm 1.6$ & $41.3 \pm 1.7$ & $41.0 \pm 1.6$ & \\
\hline Total TMIG-IC score (points) & $13.0(12.0-13.0)$ & $13.0(12.0-13.0)$ & $13.0(12.0-13.0)$ & $\mathrm{a}$ \\
\hline \multicolumn{5}{|c|}{$\begin{array}{l}\text { TMIG-IC subscales score of instrumental } \\
\text { self-maintenance, } \mathrm{n}(\%)\end{array}$} \\
\hline 5 points & $126(90.0)$ & $49(87.5)$ & 77 (91.7) & \multirow{6}{*}{$\mathrm{b}$} \\
\hline 4 points & $9(6.4)$ & $6(10.7)$ & $3(3.6)$ & \\
\hline 3 points & $4(2.9)$ & $1(1.8)$ & $3(3.6)$ & \\
\hline 2 points & $0(0)$ & $0(0)$ & $0(0)$ & \\
\hline 1 point & $1(0.7)$ & $0(0)$ & $1(1.2)$ & \\
\hline 0 point & $0(0)$ & $0(0)$ & $0(0)$ & \\
\hline
\end{tabular}

Values are median (interquartile range), number (\%), and mean \pm standard deviation. BMI: body mass index; Independent: going outside independently; House-bound: needing help to go outside but in general living independently in the house; Bed-bound: needing help for all basic activities of daily living; FIDS: Functional Independence and Difficulty Scale; BI: Barthel index; TMIGIC: Tokyo Metropolitan Institute of Gerontology Index of Competence

a: Unpaired Mann-Whitney U-test, b: $\chi^{2}$ test

$* \mathrm{p}<0.05, * * \mathrm{p}<0.01$

with and without adverse events, those who experienced adverse events showed significantly lower scores on instrumental self-maintenance and in terms of multiple falls. On the other hand, significantly lower BI scores were associated only with 
Table 2. Comparison of baseline characteristics between participants with and without IADL decline and multiple falls in the 24-month follow-up survey $(\mathrm{n}=140)$

\begin{tabular}{|c|c|c|c|c|c|c|}
\hline & \multicolumn{3}{|c|}{ IADL decline } & \multicolumn{3}{|c|}{ Multiple falls } \\
\hline & $\begin{array}{l}\text { No decline } \\
(\mathrm{n}=125)\end{array}$ & $\begin{array}{c}\text { Decline } \\
(\mathrm{n}=15)\end{array}$ & & $\begin{array}{c}\text { Non-faller } \\
(\mathrm{n}=126)\end{array}$ & $\begin{array}{l}\text { Faller } \\
(\mathrm{n}=14)\end{array}$ & \\
\hline Age (years) & $74.0(70.0-80.0)$ & $80.0(74.0-85.0)^{*}$ & $\mathrm{a}$ & $74.0(70.0-80.0)$ & $74.5(71.0-83.0)$ & $\mathrm{a}$ \\
\hline Gender (Male/Female) & $48 / 77$ & $8 / 7$ & $\mathrm{~b}$ & $47 / 79$ & $9 / 5$ & $\mathrm{~b}$ \\
\hline Height (cm) & $153.5(148.5-159.5)$ & $152.5(146.3-159.7)$ & $\mathrm{a}$ & $153.2(148.2-160.0)$ & $155.5(152.5-157.7)$ & $\mathrm{a}$ \\
\hline Weight (kg) & $56.0(48.9-62.1)$ & $55.2(48.3-63.4)$ & $\mathrm{a}$ & $55.4(48.8-62.2)$ & $57.0(50.2-62.5)$ & $\mathrm{a}$ \\
\hline BMI $\left(\mathrm{kg} / \mathrm{m}^{2}\right)$ & $23.2(21.4-25.5)$ & $24.1(22.1-24.9)$ & $\mathrm{a}$ & $23.2(21.4-25.5)$ & $23.3(22.5-25.9)$ & $\mathrm{a}$ \\
\hline BI score (points) & $100(100-100)$ & $100(95.0-100)^{*}$ & $\mathrm{a}$ & $100(100-100)$ & $100(100-100)$ & $\mathrm{a}$ \\
\hline FIDS score (points) & $42.0(41.0-42.0)$ & $40.0(38.0-42.0)^{*}$ & $\mathrm{a}$ & $42.0(41.0-42.0)$ & $41.0(39.0-42.0)^{*}$ & $\mathrm{a}$ \\
\hline
\end{tabular}

Values are median (interquartile range) and percentage (\%). IADL: instrumental activities of daily living; BMI: body mass index; BI: Barthel index; FIDS: Functional Independence and Difficulty Scale a: Unpaired Mann-Whitney U-test, $b: \chi^{2}$ test $* \mathrm{p}<0.05$

Table 3. Results of logistic regression analysis for adverse events $(n=140)$

\begin{tabular}{|c|c|c|c|c|}
\hline & \multicolumn{2}{|c|}{$\begin{array}{c}\text { IADL decline } \\
(\text { Non-decline }=0, \text { Decline }=1)\end{array}$} & \multicolumn{2}{|c|}{$\begin{array}{c}\text { Multiple falls } \\
\text { (Non-faller }=0, \text { Faller }=1)\end{array}$} \\
\hline & Model 1 & Model 2 & Model 1 & Model 2 \\
\hline \multirow[t]{2}{*}{ Model $\chi^{2}$ test } & $\mathrm{p}=0.061$ & $\mathrm{p}=0.031$ & $\mathrm{p}=0.075$ & $\mathrm{p}=0.007$ \\
\hline & ORs $(95 \% \mathrm{CI})$ & ORs $(95 \% \mathrm{CI})$ & ORs $(95 \% \mathrm{CI})$ & ORs $(95 \% \mathrm{CI})$ \\
\hline Age & $\begin{array}{c}1.063 \\
(0.972-1.162)\end{array}$ & $\begin{array}{c}1.047 \\
(0.955-1.148)\end{array}$ & $\begin{array}{c}1.010 \\
(0.921-1.107)\end{array}$ & $\begin{array}{c}0.980 \\
(0.888-1.082)\end{array}$ \\
\hline $\begin{array}{l}\text { Gender } \\
(\text { Male }=0, \text { Female }=1)\end{array}$ & $\begin{array}{c}1.661 \\
(0.529-5.213)\end{array}$ & $\begin{array}{c}1.876 \\
(0.587-5.993)\end{array}$ & $\begin{array}{c}3.184 \\
(0.954-10.627)\end{array}$ & $\begin{array}{c}3.987^{*} \\
(1.130-14.071)\end{array}$ \\
\hline $\mathrm{BI}$ & $\begin{array}{c}0.852 \\
(0.716-1.015) \\
\end{array}$ & - & $\begin{array}{c}0.846 \\
(0.701-1.021) \\
\end{array}$ & - \\
\hline FIDS & - & $\begin{array}{c}0.745^{*} \\
(0.568-0.978)\end{array}$ & - & $\begin{array}{c}0.657 * * \\
(0.489-0.883)\end{array}$ \\
\hline$\%$ correct classifications & - & 88.6 & - & 91.4 \\
\hline
\end{tabular}

Model 1 includes age, gender, and BI as independent variables; Model 2 includes age, gender, and FIDS as independent variables.

IADL: instrumental activities of daily living; ORs: odds ratio; 95\%CI: 95\% confidence interval; $\mathrm{BI}$ : Barthel index; FIDS: Functional Independence and Difficulty Scale

$* \mathrm{p}<0.05, * * \mathrm{p}<0.01$

instrumental self-maintenance.

Logistic regression results are shown for both models in Table 3. FIDS scores at baseline independently predicted subsequent decline in instrumental self-maintenance [OR: 0.745, 95\% CI (0.568-0.978); $\mathrm{p}=0.034)$ ] and multiple falls [OR: 0.657, 95\% CI (0.489-0.883); $\mathrm{p}=0.005)]$. On the other hand, model 1 with $\mathrm{BI}$ as an independent variable was non-significant in the $\chi^{2}$ test. There was no statistical evidence of associations between BI score at baseline and adverse outcomes.

\section{DISCUSSION}

The major finding of the present study was that the newly developed BADL scale, the FIDS, could predict subsequent adverse health events among community-dwelling Japanese older adults. Lower FIDS scores were associated with subsequent decline of IADL ability and multiple falls. Moreover, this predictive ability was not found in the existing BADL scale, the BI. To our knowledge, this is the first study to show the predictive validity of the FIDS for adverse health events among community-dwelling Japanese older people.

The first point of discussion is the relationship between the FIDS and subsequent decline of IADL ability. The present study showed that lower FIDS scores could be an important indicator of subsequent IADL ability decline. Generally, BADLs are self-maintenance skills, such as bathing, toileting, and dressing, whereas IADLs are also routine activities, but they are more goal oriented and involve a more complex level of neuropsychological organization as compared to BADLs. Some 
studies $^{24,25)}$ have indicated that there can be a hierarchical relationship between BADLs and IADLs, as hypothesized in Lawton's model, which postulates that human behaviors can be described as a hierarchy of domains, each of which includes a set of functions that can be ordered along a continuum ${ }^{26}$. It is interesting to note that the FIDS, which assesses BADLs, a lower-level ability, can predict the decline of IADL ability, a more complex and higher-level ability. A possible explanation for this result is that the FIDS assesses the disability regarding not only "dependency" but also "perceived difficulty," which might then capture potential subgroups at risk of subsequent IADL decline using the construct of perceived difficulty. Gill and colleagues demonstrated that "perceived difficulty associated with BADL" is an important sign of functional loss in older adults performing BADLs independently"). Moreover, among older adults who perform BADLs independently, those performing BADLs independently but with difficulty were a population at high risk for developing subsequent BADL dependence, admission to a skilled nursing facility, and death"). The FIDS, which includes assessment of "difficulty" and could capture important signs of functional loss and subsequent function decline, might predict subsequent decline of IADLs.

The second concern is the relationship between the FIDS and falls. Our results showed that lower FIDS scores could be a significant predictor of multiple falls. Although many distinct causes of falls in older people have been reported by several studies $^{27,28)}$, impaired physical function, particularly muscle weakness and problems with gait and balance, are the important contributors to the risk of falling ${ }^{29)}$. Additionally, among community-dwelling older adults, HRQOL has been reported as an independent predictor of falls ${ }^{16}$. A possible explanation for our result is that the FIDS reflects these fall-related factors. In our previous studies ${ }^{9,11)}$, the FIDS showed associations with physical functions, particularly with gait speed, balance, and HRQOL. These previous results suggest that the FIDS is related to physical function and HRQOL. The FIDS, which reflects physical function and HRQOL, might predict multiple falls in our study population.

The third point of discussion is the difference between the FIDS and BI. In this study, the FIDS was predictive of the subsequent decline of IADL ability and multiple falls. However, this predictive ability was not found for the BI. A possible explanation for this difference is that the BI could not fully capture BADL disability. Although both scales capture BADL disability, the FIDS captures BADL disability in dependency and difficulty, whereas the BI captures BADL disability in dependency only. The BI could not capture older people who perform BADLs independently with difficulty and those suspected to be at risk of adverse health events. This difference might have contributed to our results.

Our study showed that the newly developed FIDS could predict subsequent decline of IADL ability and multiple falls in community-dwelling elderly Japanese adults. These results indicated and support the prognostic relevance of the FIDS. Therefore, the FIDS might be used as a tool to screen older adults for vulnerability to subsequent IADL decline and multiple falls in community settings of Japanese older adults.

There were several limitations to the present study. First, additional studies using a sufficiently large probability sample including diverse samples of participants are needed to better examine the relationship between the FIDS and subsequent IADL decline and multiple falls. Because we used convenience sampling as our recruitment method, our study sample was small and the distribution of the baseline scores of FIDS was biased toward higher scores. Second, our study used "IADL decline" and "multiple falls" as the adverse health outcome measures, so we cannot generalize our results to other outcomes, such as institutionalization, healthcare utilization, and death. Third, we analyzed retrospectively recalled falls. This is known to be a less accurate measure than prospectively recalled falls ${ }^{30}$. It is possible that underreporting of falls by participants may have influenced the relationship between FIDS scores and multiple falls. Therefore, further studies using a monitoring system such as monthly/weekly telephone follow-ups or face-to-face interviews might be useful ${ }^{31)}$. Fourth, when we performed logistic regression analysis, age and gender were included as independent variables to adjust the relationship between the FIDS and outcome measures. However, no adjustment was made for other potential confounds, such as walking ability ${ }^{32)}$, cognitive function ${ }^{32)}$, and past history of falls ${ }^{27}$. Additional studies considering these factors as potential confounds when adjusting the relationship between the FIDS and adverse health events are needed.

In conclusion, this is the first study to show the predictive validity of the newly developed FIDS for subsequent IADL decline and multiple falls among community-dwelling Japanese older adults. It is noteworthy that this predictive ability was not found in an existing BADL scale, the BI. With additional studies, the FIDS might be used as a tool to screen older adults for vulnerability to subsequent IADL decline and multiple falls in community settings of Japanese older adults.

\section{REFERENCES}

1) Katz S, Ford AB, Moskowitz RW, et al.: Studies of illness in the aged. The index of ADL: a standardized measure of biological and psychosocial function JAMA, 1963, 185: 914-919. [Medline] [CrossRef]

2) Chei CL, Raman P, Yin ZX, et al.: Vitamin D levels and cognition in elderly adults in China. J Am Geriatr Soc, 2014, 62: 2125-2129. [Medline] [CrossRef]

3) Figueiredo CS, Assis MG, Silva SL, et al.: Functional and cognitive changes in community-dwelling elderly: longitudinal study. Braz J Phys Ther, 2013, 17: 297-306. [Medline] [CrossRef]

4) Studenski S, Duncan PW, Perera S, et al.: Daily functioning and quality of life in a randomized controlled trial of therapeutic exercise for subacute stroke survivors. Stroke, 2005, 36: 1764-1770. [Medline] [CrossRef]

5) Hirose T, Hasegawa J, Izawa S, et al.: [Investigation of whether depressive status contributes to mortality and hospitalization in community-dwelling dependent older people]. Nippon Ronen Igakkai Zasshi, 2011, 48: 163-169 (in Japanese with English abstract). [Medline] [CrossRef] 
6) Gill TM, Robison JT, Tinetti ME: Difficulty and dependence: two components of the disability continuum among community-living older persons. Ann Intern Med, 1998, 128: 96-101. [Medline] [CrossRef]

7) Gill TM: Assessment of function and disability in longitudinal studies. J Am Geriatr Soc, 2010, 58: S308-S312. [Medline] [CrossRef]

8) Saito T, Izawa KP, Omori Y, et al.: Functional independence and difficulty scale: instrument development and validity evaluation. Geriatr Gerontol Int, 2016, 16: 1127-1137. [Medline] [CrossRef]

9) Saito T, Izawa KP, Matsui N, et al.: Comparison of the measurement properties of the Functional Independence and Difficulty Scale with the Barthel Index in community-dwelling elderly people in Japan. Aging Clin Exp Res, 2017, 29: 273-281. [Medline]

10) Saito T, Izawa KP, Watanabe S: The relative and absolute reliability of the Functional Independence and Difficulty Scale in community-dwelling frail elderly Japanese people using long-term care insurance services. Aging Clin Exp Res, 2016 [Epub ahead of print]. [Medline]

11) Saito T, Izawa KP, Watanabe S: The association between the Functional Independence and Difficulty Scale and physical functions in community-dwelling Japanese older adults using long-term care services. J Geriatr Phys Ther, 2016 [Epub ahead of print]. [Medline] [CrossRef]

12) Martinsson L, Eksborg S: Activity Index - a complementary ADL scale to the Barthel Index in the acute stage in patients with severe stroke. Cerebrovasc Dis, 2006, 22: 231-239. [Medline] [CrossRef]

13) McDowell I: Measuring health: a guide to rating scales and questionnaires, 3rd ed. Oxford: Oxford University Press, 2006, pp 55-149.

14) Mahoney FI, Barthel DW: Functional evaluation: the Barthel index. Md State Med J, 1965, 14: 61-65. [Medline]

15) Guralnik JM, Ferrucci L, Simonsick EM, et al.: Lower-extremity function in persons over the age of 70 years as a predictor of subsequent disability. N Engl J Med, 1995, 332: 556-561. [Medline] [CrossRef]

16) Bilotta C, Bowling A, Nicolini P, et al.: Older People's Quality of Life (OPQOL) scores and adverse health outcomes at a one-year follow-up. A prospective cohort study on older outpatients living in the community in Italy. Health Qual Life Outcomes, 2011, 9: 72. [Medline] [CrossRef]

17) Hamilton BB, Granger CV: Disability outcomes following inpatient rehabilitation for stroke. Phys Ther, 1994, 74: 494-503. [Medline] [CrossRef]

18) Bates B, Choi JY, Duncan PW, et al. US Department of Defense Department of Veterans Affairs: Veterans Affairs/Department of Defense clinical practice guideline for the management of adult stroke rehabilitation care: executive summary. Stroke, 2005, 36: 2049-2056. [Medline] [CrossRef]

19) Wade DT, Collin C: The Barthel ADL Index: a standard measure of physical disability? Int Disabil Stud, 1988, 10: 64-67. [Medline] [CrossRef]

20) Ministry of Health, Labour and Welfare. Health and Welfare Services for the Elderly. http://www.mhlw.go.jp/english/policy/care-welfare/care-welfare-elderly/ index.html (Accessed Aug. 23, 2016)

21) Koyano W, Shibata H, Nakazato K, et al.: Measurement of competence: reliability and validity of the TMIG Index of Competence. Arch Gerontol Geriatr, 1991, 13: 103-116. [Medline] [CrossRef]

22) Martin KL, Blizzard L, Srikanth VK, et al.: Cognitive function modifies the effect of physiological function on the risk of multiple falls-a population-based study. J Gerontol A Biol Sci Med Sci, 2013, 68: 1091-1097. [Medline] [CrossRef]

23) Tinetti ME, Williams CS: The effect of falls and fall injuries on functioning in community-dwelling older persons. J Gerontol A Biol Sci Med Sci, 1998, 53: M112-M119. [Medline] [CrossRef]

24) Barberger-Gateau P, Rainville C, Letenneur L, et al.: A hierarchical model of domains of disablement in the elderly: a longitudinal approach. Disabil Rehabil, 2000, 22: 308-317. [Medline] [CrossRef]

25) Spector WD, Katz S, Murphy JB, et al.: The hierarchical relationship between activities of daily living and instrumental activities of daily living. J Chronic Dis, 1987, 40: 481-489. [Medline] [CrossRef]

26) Lawton MP: Assessing the competence of older people. In: Kent DP, Kastenbaum R, Sherwood S (eds.), Research planning and action for the elderly: the power and potential of social science. Behavioral Publications, 1972, pp 122-143.

27) American Geriatrics Society: British Geriatrics Society, and American Academy of Orthopaedic Surgeons Panel on Falls Prevention: guideline for the prevention of falls in older persons. J Am Geriatr Soc, 2001, 49: 664-672. [Medline] [CrossRef]

28) Moreland J, Richardson J, Chan D, et al.: Evidence-based guidelines for the secondary prevention of falls in older adults. Gerontology, 2003 , 49: 93-116. [Medline] [CrossRef]

29) Rubenstein LZ: Falls in older people: epidemiology, risk factors and strategies for prevention. Age Ageing, 2006, 35: ii37-ii41. [Medline] [CrossRef]

30) Cummings SR, Nevitt MC, Kidd S: Forgetting falls. The limited accuracy of recall of falls in the elderly. J Am Geriatr Soc, 1988, 36: 613-616. [Medline] [CrossRef]

31) Lamb SE, Jørstad-Stein EC, Hauer K, et al. Prevention of Falls Network Europe and Outcomes Consensus Group: Development of a common outcome data set for fall injury prevention trials: the Prevention of Falls Network Europe consensus. J Am Geriatr Soc, 2005, 53: 1618-1622. [Medline] [CrossRef]

32) Makizako H, Shimada H, Doi T, et al.: Cognitive functioning and walking speed in older adults as predictors of limitations in self-reported instrumental activity of daily living: prospective findings from the Obu Study of Health Promotion for the Elderly. Int J Environ Res Public Health, 2015, 12: 3002-3013. [Medline] [CrossRef] 